\title{
Experience shapes our odor perception but depends on the initial perceptual processing of the stimulus
}

\author{
Charlotte Sinding • Gérard Coureaud • Boris Bervialle • \\ Christophe Martin • Benoist Schaal • Thierry Thomas-Danguin
}

Published online: 2 April 2015

(C) The Psychonomic Society, Inc. 2015

\begin{abstract}
The questions of whether configural and elemental perceptions are competitive or exclusive perceptual processes and whether they rely on independent or dependent mechanisms are poorly understood. To examine these questions, we modified perceptual experience through preexposure to mixed or single odors and measured the resulting variation in the levels of configural and elemental perception of target odor mixtures. We used target mixtures that were spontaneously processed in a configural or an elemental manner. The $\mathrm{AB}$ binary mixture spontaneously involved the configural perception of a pineapple odor, whereas component A smelled like strawberry and component B smelled like caramel. The CD mixture produced the elemental perceptions of banana (C) and smoky (D) odors. Perceptual experience was manipulated through repeated exposure to either a mixture $(\mathrm{AB}$ or $\mathrm{CD})$ or the components ( $\mathrm{A}$ and $\mathrm{B}$ or $\mathrm{C}$ and $\mathrm{D}$ ). The odor typicality rating data recorded after exposure revealed different influences of experience on odor mixtures and single-component perception, depending both on the type of exposure (components or mixture) and the mixture's initial perceptual property (configural or elemental). Although preexposure to A and B decreased the pineapple typicality of the configural $\mathrm{AB}$
\end{abstract}

C. Sinding $\cdot$ G. Coureaud $\cdot$ B. Bervialle $\cdot$ C. Martin $\cdot$ B. Schaal

T. Thomas-Danguin

Centre des Sciences du Goût et de l'Alimentation, CNRS, UMR

6265, INRA, UMR 1324, Université de Bourgogne, Dijon, France

T. Thomas-Danguin $(\bowtie)$

Centre des Sciences du Goût et de l'Alimentation, INRA, Université

de Bourgogne, 17 rue Sully, 21000 Dijon, France

e-mail: Thierry.Thomas-Danguin@dijon.inra.fr

Present Address:

C. Sinding

Smell \& Taste Clinic, Department of Otorhinolaryngology, TU

Dresden, Universitätsklinikum Carls Gustav Carus,

Dresden, Germany mixture, preexposure to $\mathrm{AB}$ did not modify its odor quality. In contrast, preexposure to the $\mathrm{CD}$ elemental mixture induced a quality transfer between the components. These results emphasize the relative plasticity of odor mixture perception, which is prone to experience-induced modulations but depends on the stimulus's initial perceptual properties, suggesting that configural and elemental forms of odor mixture perception rely on rather independent processes.

Keywords Odor mixture $\cdot$ Blending $\cdot$ Configural . Elemental $\cdot$ Perceptual experience

The myriad of odorants that we encounter in our daily life (e.g., food, surroundings, social odors, or perfumes) give rise to a variety of percepts, which may vary according to contextual and individual factors. In humans, interindividual differences in odor perception have been found to be associated not only with age (Cain \& Gent, 1991; Thomas-Danguin et al., 2003) and health status (Doty, 2001), but also with cognitive factors (Chrea et al., 2004) and semantic knowledge (Rouby et al., 2005). These last two factors underline the critical role of memory in odor processing (Wilson \& Stevenson, 2003). As in nearly all sensory systems, olfactory perceptual learning contributes to improving our ability to discriminate between odors and categorize them. However, odors are hardly ever perceived alone in real life, and the olfactory system must be efficient to analyze mixtures of tens of odorants. To do so, configural (synthetic) and elemental (analytic) processes can be engaged, which dramatically increases the variety of percepts that can be perceived (Bushdid, Magnasco, Vosshall, \& Keller, 2014). Elemental processing of odor mixtures allows recognition of the odors of the components, up to a maximum of four in humans (Livermore \& Laing, 1998; Livermore \& Laing, 1996). Configural processing of odor mixtures gives rise to the perception of a new odor, specific to the 
configuration and distinct from the odors of the components (Kay, Crk, \& Thorngate, 2005). Thus, configural perception contributes to the object recognition functioning of olfaction (Stevenson \& Wilson, 2007; Thomas-Danguin et al., 2014).

Even though the role of experience in odor processing remains a central issue in olfactory research, only a few studies have dealt with the impact of perceptual experience on odor mixture perception. Mixture perception relies on two aspects: the abilities to recognize components within mixtures (elemental perception) and to process mixtures as single odor objects (configural perception; e.g., Coureaud, ThomasDanguin, Wilson, \& Ferreira, 2014; Livermore, Hutson, Ngo, Hadjisimos, \& Derby, 1997; Rabin, 1988; Sinding, Thomas-Danguin, Crepeaux, Schaal, \& Coureaud, 2011). Most animal studies have used conditioning to show the effects of experience on odor mixture perception. Indeed, after repeated conditioning to binary or ternary mixtures initially perceived in an elemental manner, tested rats or catfishes no longer generalized the learned information to its single components, suggesting that the mixtures became perceived as configurations (Staubli, Fraser, Faraday, \& Lynch, 1987; Valentincic, Kralj, Stenovec, Koce, \& Caprio, 2000). In newborn rabbits, it has been shown that repeated conditioning can favor configural processing of the AB mixture (ethyl isobutyrate + ethyl maltol; Sinding et al., 2011) known to elicit the configural perception of a pineapple odor in human adults (Le Berre et al., 2010; Le Berre et al., 2008). Indeed, after a single conditioning to one component of the mixture, 2-dayold rabbit pups selectively responded to that odorant, but they did not respond to the $\mathrm{AB}$ mixture. In contrast, after a single conditioning to the mixture, they responded both to the mixture and to the component odorants. These results illustrate the weak configural perception of the $\mathrm{AB}$ mixture by rabbit neonates (at a specific 30/70 ratio v/v of A and B; Coureaud, Gibaud, Le Berre, Schaal, \& Thomas-Danguin, 2011; Coureaud, Hamdani, Schaal, \& Thomas-Danguin, 2009; Coureaud, Thomas-Danguin, Le Berre, \& Schaal, 2008; Coureaud et al., 2014). However, after repeated conditioning (three episodes) to one component, a significant proportion of pups became able to perceive the learned element within the AB mixture. Conversely, repeated conditioning (nine episodes) to the AB mixture improved its configural perception, because a large percentage of pups responded to the mixture, but only a few responded to the single components (Sinding et al., 2011). Such experience-induced improvements of elemental or configural perception were not observed with an AC mixture, which remained elementally processed by rabbit pups, regardless of their experience with the mixture or its components (Coureaud et al., 2009; Sinding et al., 2011). Overall, these results suggest that experience could modulate the perception of some, but not all, odor mixtures.

Neurophysiological correlates of such processes have been shown in rats without a conditioning design. Once exposed for
10 or $50 \mathrm{~s}$ to a binary mixture to induce habituation (i.e., decreased response interest toward the odor), free-breathing anesthetized rats were stimulated to one component while the olfactory bulb and the anterior piriform cortex were recorded with a single electrode. Only the rats exposed to the mixture for $50 \mathrm{~s}$ (a longer experience of the mixture) did not present cross-habituation to the single components (i.e., they did not generalize between the mixture and its components; Wilson, 2003). Supporting the behavioral data, the electrophysiological results highlighted that the neurons recorded in the anterior piriform cortex were still responding to the single components, although they did not respond to the mixture anymore (Wilson, 2003).

In humans, it has been shown that odors experienced in mixtures transfer part of their respective odor qualities to other odors in the mixture (Stevenson, 2001c). Indeed, subjects who were exposed to binary odor mixtures (mint + cherry or smoky + lemon) and who later evaluated the respective qualities of the components and mixtures perceived a cherry odor in the mint component and a smoky odor in the lemon one (Stevenson, 2001a). This transfer of qualities between paired odorants has been interpreted as a form of association between the mixture's elements, in line with configural processing of the mixtures that is induced by coexposure and learning (Case, Stevenson, \& Dempsey, 2004; Stevenson, 2001a). Conversely, in another study, subjects were exposed to the components of a ternary mixture that initially elicited the perception of a pineapple odor. Then they were asked to rate the mixture's pineapple odor quality, which was found to be lower for subjects previously exposed to the components than for the subjects previously exposed to unrelated odors. In that case, exposure to the elements seemed to reduce configural processing of the mixture as the result of an experience-induced enhancement of the components' salience (Le Berre et al., 2008).

The effect of perceptual learning of either a mixture or its components on the subsequent processing of the mixture as a single object or as a collection of odors remains poorly understood. Such an impact of experience on odor perception is critical, because in ecological conditions, odorants are encountered in various overlapping mixtures, and their categorization and discrimination are shaped by experience. A primary reason for the lack of knowledge about experiential modulation of odor mixture perception is that these processes are driven by various factors, such as the chemical nature of the mixed odorants and their proportions, as well as individual cognitive factors (Thomas-Danguin et al., 2014). A second reason is that configural and elemental processes are overlapping and most likely competitive mechanisms (ThomasDanguin et al., 2014). Thus, to fully understand these mechanisms, we have to consider their possible interactions and dependencies, and therefore we need to test the modulation of both configural and elemental perception of the same odor mixture with identical experience levels. We report here a 
study that covered different aspects of experience for both configural and elemental perceptions. We set out to determine how the perception of simple odor mixture models (two binary mixtures) could vary according to experience with either the mixture or its components. The achievement of this goal was facilitated by the use of a specific odor model - a blending mixture-that can easily be tested for experiencedependent modulation of configural and elemental perceptions. We assessed whether repeated exposure to either a mixture or its single components influenced the later perception of these stimuli, depending on whether or not the mixture had blending properties (i.e., gave rise to a configural perception). The previously mentioned $\mathrm{AB}$ blending mixture (pineapple odor; Barkat et al., 2012; Le Berre et al., 2010; Le Berre et al., 2008) was used, as well as another binary mixture (CD) formulated to be a nonblending mixture. Four groups of subjects were repeatedly (over two sessions) preexposed to the $\mathrm{AB}$ or the $\mathrm{CD}$ mixture, or to their $\mathrm{A}$ and $\mathrm{B}$ or $\mathrm{C}$ and $\mathrm{D}$ single components. Then, subjects were tested for their perception of all of the stimuli (mixtures and single components) using a typicality rating test (Le Berre et al., 2008). More precisely, our first two hypotheses were that preexposure to the components A and B (i.e., exposure to the odors before testing to induce perceptual experience) should result in reduced configural perception of the $\mathrm{AB}$ blending mixture (i.e., a decrease in pineapple odor typicality), whereas preexposure to the mixture would favor its configural perception (i.e., increase its "pineappleness"). The third and fourth hypotheses were that preexposure to the nonblending $\mathrm{CD}$ mixture should induce configural processing of this mixture, which could be observed by a decrease in the individual component qualities (typicality) within the mixture, whereas preexposure to the $\mathrm{C}$ and $\mathrm{D}$ single components could increase their salience in the CD mixture.

\section{Materials and method}

\section{Odor stimuli}

All of the chemicals were of food-grade quality and were purchased from Sigma-Aldrich (Saint-Quentin Fallavier, France), except where indicated.

\section{Preliminary study}

We set out to find which concentrations of odorants allowed for the balanced perception of the odors carried by odorants $\mathrm{C}$ (guaiacol, CAS \# 90-05-1, smoky odor) and D (isoamyl acetate, CAS \# 123-92-2, banana odor) in the CD nonblending mixture (on the basis of Atanasova et al., 2005). We also checked for the appropriateness of the terms that described the odors of C (smoky) and D (banana). Stock solutions of single odorants were prepared as follows: $83 \% \mathrm{w} / \mathrm{w}$ ratio of D in ethanol (99 \% anhydrous, European Pharmacopoeia grade; Carlos Erba, Val de Reuil, France), whereas $\mathrm{C}$ was used pure $(100 \%)$. To determine the ratio of $\mathrm{C} / \mathrm{D}$ that corresponds optimally to a balanced quality and intensity of the two odors, we used six distinct $\mathrm{w} / \mathrm{w}$ ratios of $\mathrm{C} / \mathrm{D}$ in mixture $(100 / 0,87.5 / 12.5$, $81.25 / 18.75,75 / 25,60 / 40$, and $0 / 100)$. These odor stimuli were all presented on paper strips (Granger-Veyron, France) and placed in $60-\mathrm{mL}$ brown glass vials (see below). Each vial contained four strips of paper, each receiving $20 \mu \mathrm{L}$ of solution (single odorant or mixture). Vials were prepared the day before the experiment and stored in the air-conditioned room $\left(21^{\circ} \mathrm{C}\right)$ where the sensory experiments were carried out. As a result, the 40/60 w/w C/D solution was selected for the main study (see the Results, Preliminary Study section).

\section{Main study}

The stimuli used were odorants $\mathrm{C}$ and $\mathrm{D}$, the $\mathrm{CD}$ nonblending mixture (see the preliminary study), odorants A (ethyl isobutyrate, CAS \# 97-62-1, strawberry odor) and B (ethyl maltol, CAS \# 4940-11-8, caramel odor), and the AB blending mixture, known to have a pineapple configural odor (Le Berre et al., 2008). Stock solutions of the single odorants A and B were prepared as follows: $10 \% \mathrm{w} / \mathrm{w}$ ratio of $\mathrm{A}$ in ethanol, $1 \%$ $\mathrm{w} / \mathrm{w}$ ratio of $\mathrm{B}$ in ethanol, and the $\mathrm{AB}$ mixture at a $30 / 70 \mathrm{v} / \mathrm{v}$ ratio of the $\mathrm{A}$ and $\mathrm{B}$ stock solutions, respectively. The two mixtures and their single components were used during the preexposure sessions and tests (same concentrations).

During preexposure sessions, four odor stimuli were used as distracters to prevent subjects from focusing on the target odors (i.e., the exposure should induce as little cognitive processing as possible): two complex mixtures that smelled like hyacinth ( $\mathrm{H}$, a six-component mixture designed by a flavorist) and chive (Ch, a five-component mixture designed by a flavorist), and two single odorants L (D-limonene, CAS \# 598927-5, citrus odor) and I (isoeugenol, CAS \# 97-54-1, carnation odor). Another blending mixture, RC (six components, smelling like red cordial, also named grenadine; Le Berre et al., 2008; Sinding et al., 2013) was used as a control stimulus during testing but not during the preexposure sessions.

The odor stimuli were all presented on paper strips placed in Teflon bags. This method was chosen because of the temporal stability of the gaseous-phase composition (Atanasova, Langlois, Nicklaus, Chabanet, \& Etiévant, 2004). Each bag contained four paper strips, each spiked with $20 \mu \mathrm{L}$ of solution (single odorant or mixture). The strips were placed for $2 \mathrm{~min}$ under an extractor hood to evaporate the ethanol before they were introduced into the bags, which were then filled with $17 \mathrm{~L}$ of pure nitrogen. The bags were prepared the day before the first preexposure and were kept during the week of exposure in the air-conditioned room $\left(21^{\circ} \mathrm{C}\right)$ where the sensory assays occurred. 
Subjects

Twenty-one subjects ( 15 women and 6 men; mean age: $27.5 \pm$ 7.8 years), all members of the laboratory (CSGA-Dijon), participated in the preliminary experiment.

In the main study, 78 subjects (51 women and 27 men; mean age: $39.9 \pm 14.6$ years) were first selected from a pool of 83 candidates (general population of Dijon and surroundings) on the basis of their (1) olfactory abilities, evaluated with the European Test of Olfactory Capabilities (ETOC; ThomasDanguin et al., 2003); (2) semantic abilities in relation to odor (free-descriptions task; Sulmont-Rossé, Issanchou, \& Köster, 2005); and (3) mental concentration ability (Bourdon test; Lesschaeve \& Issanchou, 1996). The total scores on these tests ranged from $41 \%$ to $93 \%$ for the whole panel. Five candidates were not selected because their scores were lower than $60 \%$. The 78 remaining subjects were randomly allocated to four groups of 19 or 20.

The subjects were considered "naïve" because they had no special expertise in olfaction or sensory analysis. They were recruited with no self-reported sense of smell problems or allergies. Selected panelists signed a consent form but were not informed of the aim of the study. They received compensation of $€ 10$ for each hour of experimentation. The experiments were carried out in accordance with the Declaration of Helsinki and took into consideration French laws and regulations (Huriet-Sérusclat Law, France, 1988).

\section{Experimental procedure}

\section{Preliminary study}

First, to check for the appropriateness of the odor descriptors, subjects had to describe the odors of odorants $\mathrm{C}$ and $\mathrm{D}$ using a free-description procedure. The terms most frequently cited were confirmed to be smoky and banana for C and D, respectively. Second, they evaluated, in a counterbalanced order across subjects, the intensities of these smoky and banana odors in six solutions that varied in terms of $\mathrm{C} / \mathrm{D}$ ratio. Intensity was rated on two 10 -cm linear scales, one labeled "smoky odor" and the other labeled "banana odor," with no graduations but end point anchors of weak and strong.

\section{Main study}

Preexposure Prior to the test session, the four groups of subjects were exposed to different target stimuli presented among distracting ones $(\mathrm{H}, \mathrm{Ch}, \mathrm{L}$, and I). Group $1(\mathrm{G} 1, N=20)$ was preexposed to the AB blending mixture, Group $2(\mathrm{G} 2, N=19)$ to the A and B components out of mixture, Group $3(\mathrm{G} 3, N=$ 19 ) to the selected CD nonblending mixture, and Group 4 (G4, $N=20$ ) to the unmixed components $\mathrm{C}$ and D.
The preexposure consisted of two identical sessions separated by a week (Fig. 1). To keep subjects blind to the aim of the experiment, they were asked to evaluate their general mood using the Differential Emotion Scale (Ouss, Carton, Jouvent, \& Widlöcher, 1990) and the Self-Assessment Manikin (Morris, 1995) while they were smelling each of the five $(\mathrm{G} 1, \mathrm{G} 3)$ or six $(\mathrm{G} 2, \mathrm{G} 4)$ odor stimuli. A total of 11 items were rated, corresponding to a total of at least 11 sniffs of each stimulus per session. The presentation order of the stimuli was counterbalanced across subjects.

Test session One week after the last preexposure session, all subjects evaluated the $\mathrm{AB}$ and $\mathrm{CD}$ target mixtures and their single components. Subjects performed a typicality rating task (Barkat, Le Berre, Coureaud, Sicard, \& Thomas-Danguin, 2012; Le Berre et al., 2010; Le Berre et al., 2008) for each stimulus, responding to the question: "Is this odor a good or a poor example of the odor of X?," where X could be "pineapple," "smoky," "banana," or "red cordial." Subjects rated the pineapple typicality of $\mathrm{AB}, \mathrm{A}$, and B; the smoky or banana typicality of CD, C, and D; and the red cordial typicality of RC on a 10-cm linear scale (end point anchors: poor example-good example).

Eight Teflon bags, each containing one stimulus, were evaluated by the subjects in a specific order (Fig. 1): RC first; $\mathrm{AB}$ and $\mathrm{CD}$ in a counterbalanced order across subjects; then $\mathrm{A}, \mathrm{B}, \mathrm{C}$, and $\mathrm{D}$; and finally $\mathrm{RC}$ again. The mixtures were always evaluated before components, to avoid elemental processing due to prior exposure to the components (Le Berre et al., 2008). All of the groups of subjects evaluated all of the stimuli during the test session. For the stimuli $\mathrm{CD}, \mathrm{C}$, and $\mathrm{D}$, each group was separated into two subgroups; one evaluated the smoky typicality, and the other evaluated the banana typicality. Repeated evaluations of RC were run in order to evaluate subjects' response consistency.

\section{Statistical analysis}

Sensory data were recorded with FIZZ software (Biosystèmes, Couternon, France). Statistical analyses were conducted using R software (version 2.10.1; R Foundation for Statistical Computing, Vienna, Austria). Because of low numbers of subjects in some groups ( $n=10$ for the smallest group), we used nonparametric tests. Although Wilcoxon rank sum tests are usually applied as nonparametric two-sample tests, they are not recommended due to their strict assumptions and nonrobustness to unequal variances (Brunner \& Munzel, 2000; Neuhäuser \& Ruxton, 2009). Thus, we used the Brunner-Munzel test for two independent samples to compare the subjects' responses between groups or subgroups for the same stimulus (unpaired data, $W$ ). The Brunner-Munzel test is implemented in the R package "lawstat." The Munzel test for the stochastic equality of two dependent samples was applied in order to compare responses between stimuli within a group of subjects (paired data, $w$ ). The 


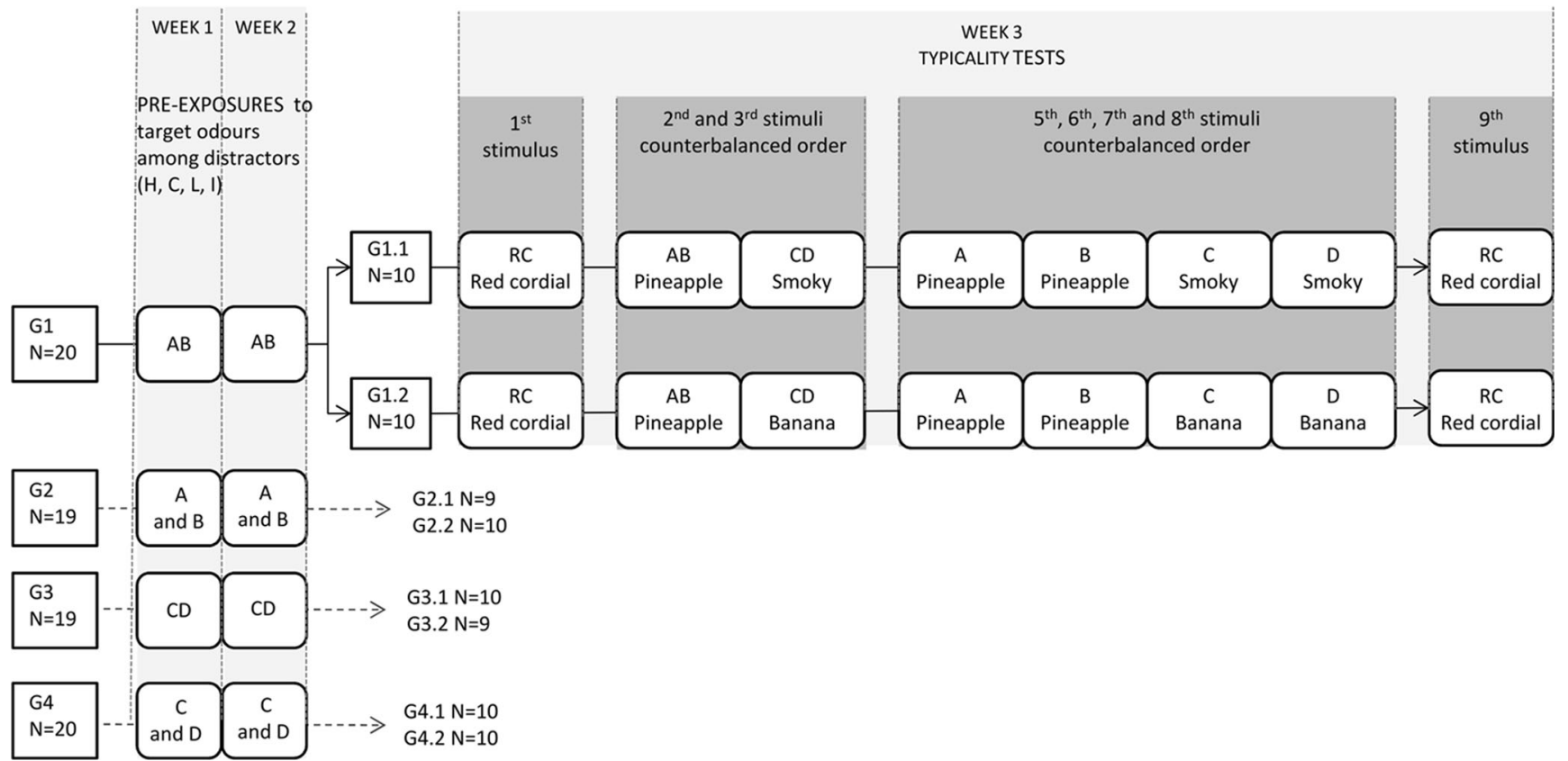

Fig. 1 Outline of the experimental procedure illustrating the stages of preexposure and testing in the G1 group and only the stage of preexposure in the G2, G3, and G4 groups, since the test session was identical for all groups. $N$ represents the number of subjects in each group or subgroup. The stimuli used during preexposure were the four distractors $(\mathrm{H}, \mathrm{Ch}, \mathrm{L}$, and $\mathrm{I})$ and, depending on the group, the pineapple blending mixture $\mathrm{AB}$, its components $\mathrm{A}$ (ethyl isobutyrate) and $\mathrm{B}$ (ethyl

$\mathrm{R}$ code for the Munzel test is given in Appendix A of Marmolejo-Ramos, Elosúa, Yamada, Hamm, and Noguchi (2013). Medians (Mdn) were calculated for each stimulus in each group. The level of significance was fixed at $p=.05$; a trend was considered when $p$ ranged between .05 and .10 . In order to ascertain a trend, a measure of stochastic superiority was used as an estimator of effect size (ES). This measure of stochastic superiority between two samples estimates the probability that a score randomly taken from the first sample will be greater than a score randomly taken from the second sample. The magnitude of the difference can be interpreted on a scale ranging from .5 to 1 , with a probability ranging from .56 to .64 reflecting a small effect size, a probability ranging from .64 to .71 reflecting a medium effect size, and a probability higher than .71 reflecting a large effect size (Marmolejo-Ramos et al., 2013). The R code used to compute the effect size for dependent samples (ES) and independent samples (es) can be found in Appendix A of Marmolejo-Ramos et al. (2013).

\section{Results}

Preliminary study: selection of odorant ratio inducing elemental perception of the $\mathrm{CD}$ mixture

To compare the effects of experience on the perceptions of the blending $(\mathrm{AB})$ or the nonblending $(\mathrm{CD})$ mixture and their maltol), the nonblending mixture $\mathrm{CD}$, or its components $\mathrm{C}$ (ethyl maltol) and D (isoamyl acetate). During the phase of testing, the stimuli for all groups were the mixtures $\mathrm{RC}, \mathrm{AB}$, and $\mathrm{CD}$ and the single components $\mathrm{A}$, $\mathrm{B}, \mathrm{C}$, and $\mathrm{D}$. In the test session, each group was subdivided in two subgroups (as G1.1 and G1.2 from G1), and the subjects in each subgroup had to rate the smoky and banana typicalities of $\mathrm{CD}, \mathrm{C}$, and $\mathrm{D}$, respectively

respective components, we first looked for the proportions of odorants $\mathrm{C}$ and $\mathrm{D}$ that induced a balanced perception of both odorants in the mixture - namely, similar intensities of C and D odor qualities. In the internal panel describing the odor quality of each odorant, the terms most frequently used were "smoky" (24 \%) and "banana" (23\%) for C and D, respectively, thus confirming the appropriateness of these two descriptors for the main study. When evaluating several CD mixtures varying in $\mathrm{C} / \mathrm{D}$ ratio $(0 / 100,12.5 / 87.5,18.75 /$ $81.25,25 / 75,40 / 60$, and 100/0) and rating smoky and banana odor intensities, the panel displayed differences between the odors, especially in samples including $100 \%$ of one odorant. Similarly, the smoky and banana odors had different intensities in the 12.5/87.5 and 18.75/81.25 ratios. However, no significant difference appeared in the $25 / 75$ or $40 / 60$ ratios $(w<$ $1.7, p>.1)$. Therefore, we decided to keep the $40 / 60 \mathrm{v} / \mathrm{v}$ ratio in the main study because it triggered the closest intensity values for banana and smoky odors within the $\mathrm{CD}$ mixture $(w=0.42, p=.68, \mathrm{ES}=0.53)$.

Main study: effects of preexposure on mixture or component perception

Confirmation of the blending effect in the AB mixture To assess the perceptual blending effect of the $\mathrm{AB}$ mixture in our experimental conditions, we used the data obtained from G3 and G4 (i.e., the groups that were not preexposed to A and B 
either in mixture or separately). The subjects in these groups rated $\mathrm{AB}$ as being more typical of the pineapple odor than either $\mathrm{A}$ or $\mathrm{B}$ alone $(w>-3.4, d f=38, p<.001$, ES $>.67$; see Tables 2 and 3 below for the median values of the pineapple typicality ratings). These results confirmed the blending property of the AB mixture-namely, its configural perception-because the mixture had an odor quality that was significantly more typical of a pineapple odor than of the single components.

Effect of preexposure on the perceptions of the AB blending mixture and its components Our first hypothesis was that preexposure to the single odorants A and B could decrease the configural perception of the $\mathrm{AB}$ mixture (i.e., favor its elemental perception). We supposed that this shift could be observed through a decrease in the typicality rating of the pineapple configural odor specific to the mixture. Our second hypothesis was that, conversely, preexposure to the AB mixture could favor its configural perception, which might be experimentally observed through an increase in the typicality rating of the pineapple odor of that mixture.

In line with our first hypothesis, the pineapple typicality of the $\mathrm{AB}$ mixture rated by $\mathrm{G} 2$ subjects (preexposed to odorants A and B) was lower than that rated by the three other groups (Fig. 2). The decrease in typicality was significant in G2 as compared with G4 ( $p=.046)$, but with a small effect size (ES $=.59$ ). However, the decrease in pineapple typicality did not

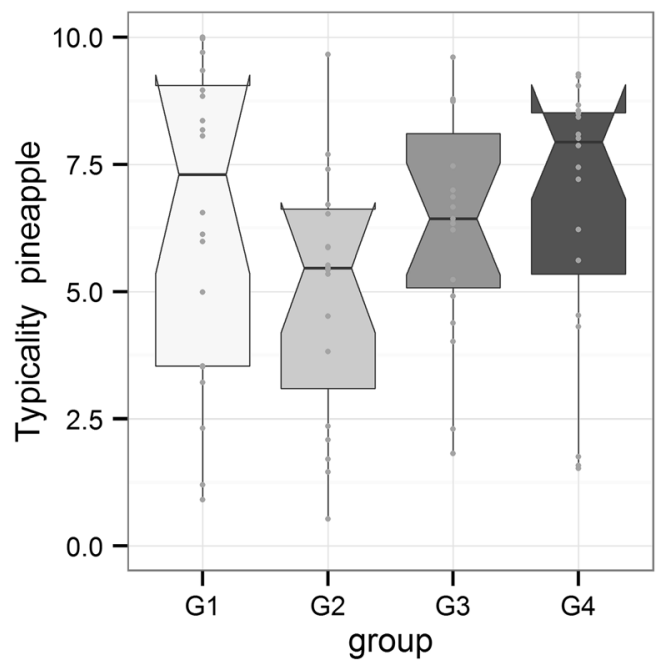

Fig. 2 Pineapple odor typicality ratings of the $A B$ mixture, composed of the odorants A (ethyl isobutyrate) and B (ethyl maltol), evaluated by subjects preexposed to $\mathrm{AB}$ (Group $1, \mathrm{G} 1 ; N=20)$, A and B $(\mathrm{G} 2 ; N=$ $19), \mathrm{CD}(\mathrm{G} 3 ; N=19)$, or $\mathrm{C}$ and $\mathrm{D}(\mathrm{G} 4 ; N=20)$. The two darker gray groups were considered controls in this analysis, because they had not been exposed to A, B, or AB. The black lines inside the boxes represent the medians. The upper and lower boxes represent the $75 \%$ and $25 \%$ quartiles. Dotted lines over the boxes range from a maximum of 1.5 times the interquartile distance, and notches represent $95 \%$ confidence intervals for the medians. Gray dots represent the individual ratings of subjects reach the significance level in $\mathrm{G} 2$ as compared with $\mathrm{G} 1$, preexposed to the $\mathrm{AB}$ mixture $(p=.12, \mathrm{ES}=.53$; see Table 1). Thus, preexposure to the single components $\mathrm{A}$ and $B$ reduced the pineapple typicality of the $A B$ mixture. In contrast with our second hypothesis, subjects preexposed to the $\mathrm{AB}$ mixture did not evaluate the $\mathrm{AB}$ mixture as being significantly more typical of pineapple than did those preexposed to CD (G1 vs. G3, $p=.65$; Table 1).

It must be noted that the pineapple typicality of odorants $A$ and $B$ remained relatively low, regardless of the group (Table 2). Moreover, the pineapple typicality of the AB mixture was higher than that of its components, regardless of the preexposure condition, in $\mathrm{G} 1, \mathrm{G} 2$, and G4 ( $p<.04$, ES > .68; see Tables 2 and 3 for the medians). Overall, these results indicate that the configural processing of the $\mathrm{AB}$ blending mixture was maintained after repeated exposures to $A B$.

Effect of preexposure on perceptions of the CD nonblending mixture and its components As with the $\mathrm{AB}$ blending mixture, we tested the hypothesis that preexposure to $\mathrm{CD}$ or to its single components could later influence the perception of these stimuli. We hypothesized that the elemental perception of $\mathrm{CD}$ could be improved after previous exposures to components $\mathrm{C}$ and $\mathrm{D}$, which might be assessed through an increase in the smoky or banana odor typicality of the mixture. However, this effect did not appear: The smoky and banana typicality of the CD mixture remained undifferentiated after preexposure to the single odorants $\mathrm{C}$ and $\mathrm{D}$, as compared with preexposure to A and B (G4 vs. G2, $p=.24$; Table 3, Fig. 3).

In contrast, we predicted an improved configural perception of $\mathrm{CD}$ after preexposure to this mixture, as reflected by a decrease in the smoky and banana odor qualities carried by $\mathrm{C}$ and $\mathrm{D}$, respectively. In other words, we expected that subjects preexposed to the CD mixture (G3) would rate the smoky or banana odor typicality as being lower in the $\mathrm{CD}$ mixture than would subjects preexposed to a distinct mixture, the AB mixture (G1). However, the results indicated that subjects in both groups did not rate the smoky odor of CD differently ( $p=.26$; Table 3 ), but G3 subjects evaluated the CD mixture as being more typical of the banana odor than did G1 subjects ( $p=.08, \mathrm{ES}=.76$; Table 3 ). In addition, whereas G2 and G4 subjects, preexposed to the single odorants $\mathrm{A}$ and $\mathrm{B}$ and the single odorants $\mathrm{C}$ and $\mathrm{D}$, respectively, gave similar smoky and banana typicality ratings to the $\mathrm{CD}$ mixture, G3 subjects, who were preexposed to the CD mixture, evaluated this mixture as being more typical of banana than did G4 subjects ( $p=.03, \mathrm{ES}=.79$; Table 3 ). Thus, only preexposure to the $\mathrm{CD}$ mixture induced a modification of the mixture perception, leading to higher banana odor typicality than smoky odor typicality. 
Table 1 Median $(M d n)$ values of pineapple typicality rating of the $\mathrm{AB}$ mixture evaluated by subjects preexposed to $\mathrm{AB}$ (Group $1, \mathrm{G} 1 ; N=20), \mathrm{A}$ and $\mathrm{B}(\mathrm{G} 2 ; N=19), \mathrm{CD}(\mathrm{G} 3 ; N=19)$, or $\mathrm{C}$ and $\mathrm{D}(\mathrm{G} 4 ; N=20)$

\begin{tabular}{|l|l|l|l|l|}
\hline $\mathbf{A B}$ & $\mathbf{G 1}(\mathrm{AB})$ & $\mathbf{G 2}(\mathrm{A} \& \mathrm{~B})$ & $\mathbf{G 3}(\mathrm{CD})$ & $\mathbf{G 4}(\mathrm{C} \& \mathrm{D})$ \\
\hline $\mathbf{G 1}(\mathrm{AB})$ & $M d n=7.3$ & & & \\
\hline $\mathbf{G} 2(\mathrm{~A} \& \mathrm{~B})$ & $\begin{array}{l}W=-1.60 \mathrm{df}=31.98 \\
p=0.12 \mathrm{ES}=0.53\end{array}$ & $M d n=5.5$ & & \\
\hline $\mathbf{G 3}(\mathrm{CD})$ & $\begin{array}{l}W=-0.46 \mathrm{df}=27.21 \\
p=0.65 \mathrm{ES}=0.59\end{array}$ & $\begin{array}{l}W=-2.07 \mathrm{df}=36.91 \\
p=0.046 \mathrm{ES}=0.59\end{array}$ & & Mdn=7.4 \\
\hline $\mathbf{G 4}(\mathrm{C} \& D)$ & & & & \\
\hline
\end{tabular}

Medians were compared with the Brunner-Munzel test for independent samples $(W:$ parameter of the Brunner-Munzel test, $d f$ : degrees of freedom, $p: p$ value. ES represents the effect size estimated through a measure of stochastic superiority $(.56 \leq$ small ES $<.64, .64 \leq$ medium ES $<.71, .71 \leq$ large ES; see the Materials and method section)

Preexposure to the CD mixture did not modify the smoky and banana typicalities of components $\mathrm{C}$ and $\mathrm{D}$, respectively, as compared with preexposure to $\mathrm{AB}$ (G3 vs. G1; Table 4, Fig. 4). Consequently, preexposure did not influence the later perception of the odorants $\mathrm{C}$ and $\mathrm{D}$ when they were evaluated separately (i.e., out of mixture).

Interestingly, a significant effect of preexposure was observed for G3 subjects (preexposed to $\mathrm{CD}$ ) when they later rated the nonrelated odor qualities of the single components. Indeed, preexposure to $\mathrm{CD}$ increased the banana typicality of the smoky-smelling odorant $\mathrm{C}$ as compared with preexposure to odorants $\mathrm{C}$ and $\mathrm{D}$ (for comparisons of G3 vs. G4 and G3 vs. $\mathrm{G} 2: p \mathrm{~s}=.02$, ESs $=.71$; Table 5, Fig. 5). A similar trend, supported by a medium effect size, appeared for G3 as compared with $\mathrm{G} 1$ subjects, preexposed to the $\mathrm{AB}$ mixture $(p=$
$.08, \mathrm{ES}=.66$; Table 5, Fig. 5). The reverse effect relative to the smoky quality of the banana-smelling odorant $\mathrm{D}$ was never noted. Thus, preexposure to the CD mixture conferred a banana odor to the smoky-smelling odorant, but not the reverse.

\section{Discussion}

In this study, we aimed to examine whether configural and elemental processing of two simple models of binary odor mixtures are modulated by experience. More precisely, we investigated the influence of prior perceptual experience with either single odor components or their mixture on the configural or elemental perception of blending $(\mathrm{AB})$ or nonblending $(\mathrm{CD})$ binary mixtures and on the perception of

Table 2 Median (Mdn) values of pineapple typicality rating of the odorants A (above the diagonal) and B (below the diagonal), evaluated by subjects preexposed to AB (Group $1, \mathrm{G} 1 ; N=20)$, A and B (G2; $N=19), \mathrm{CD}(\mathrm{G} 3 ; N=19)$, or C and D (G4; $N=20)$

\begin{tabular}{|c|c|c|c|c|}
\hline$B$ & G1 (AB) & $\mathbf{G} 2(\mathrm{~A} \& \mathrm{~B})$ & G3 (CD) & G4 (C\&D) \\
\hline $\mathbf{G 1}(\mathrm{AB})$ & $M d n=2.9$ & $\begin{array}{l}W=0.66 \mathrm{df}=35.03 \\
p=0.51 \mathrm{ES}=0.54\end{array}$ & $\begin{array}{l}W=-0.68 \mathrm{df}=36.70 \\
p=0.50 \quad \mathrm{ES}=0.59\end{array}$ & \\
\hline G2 (A\&B) & $\begin{array}{l}W=0.30 \mathrm{df}=36.80 \\
p=0.76 \mathrm{ES}=0.50\end{array}$ & $M d n=2.3$ & & $\begin{array}{l}W=1.28 \quad \mathrm{df}=34.19 \\
p=0.21 \mathrm{ES}=0.59\end{array}$ \\
\hline $\mathbf{G 3}$ (CD) & $\begin{array}{l}W=-1.38 \mathrm{df}=35.83 \\
p=0.18 \mathrm{ES}=0.59\end{array}$ & & $M d n=5.1$ & \\
\hline G4 (C\&D) & & $\begin{array}{l}W=1.16 \quad \mathrm{df}=36.78 \\
p=0.26 \mathrm{ES}=0.58\end{array}$ & & $M d n=5.2$ \\
\hline
\end{tabular}

Groups were compared with the Brunner-Munzel test for independent samples $(W$ : parameter of the Brunner-Munzel test, $d f$ : degrees of freedom, $p: p$ value). ES represents the effect size estimated through a measure of stochastic superiority $(.56 \leq$ small $\mathrm{ES}<.64, .64 \leq$ medium ES $<.71, .71 \leq$ large ES; see the Materials and method section). 
Table 3 Median (Mdn) values of smoky (above the diagonal) and banana (below the diagonal) typicality ratings of the $\mathrm{CD}$ mixture composed of C (guaiacol) and D (vanillin), evaluated by subjects preexposed to $\mathrm{AB}$ (Group $1, \mathrm{G} 1 ; N=20), \mathrm{A}$ and $\mathrm{B}(\mathrm{G} 2 ; N=19), \mathrm{CD}$ $(\mathrm{G} 3 ; N=19)$, or $\mathrm{C}$ and $\mathrm{D}(\mathrm{G} 4 ; N=20)$

\begin{tabular}{|c|c|c|c|c|}
\hline CD banana & $\mathbf{G 1}(\mathrm{AB})$ & G2 (A\&B) & G3 (CD) & G4 (C\&D) \\
\hline $\mathbf{G 1}(\mathrm{AB})$ & $M d n=2.66$ & & $\begin{array}{l}W=1.19 \mathrm{df}=12.64 \\
p=0.26 \quad \mathrm{ES}=0.66\end{array}$ & \\
\hline $\mathbf{G} 2(A \& B)$ & & $M d n=1.36$ & & $\begin{array}{l}W=0.22 \mathrm{df}=13.60 \\
p=0.83 \mathrm{ES}=0.58\end{array}$ \\
\hline $\mathbf{G} 3$ (CD) & $\begin{array}{l}W=1.89 \mathrm{df}=13.57 \\
p=0.08 \mathrm{ES}=0.76\end{array}$ & & $M d n=5.85$ & $\begin{array}{l}W=-0.43 \quad \mathrm{df}=17.76 \\
p=0.67 \quad \mathrm{ES}=0.56\end{array}$ \\
\hline G4 (C\&D) & & $\begin{array}{l}W=-1.21 \text { df }=17.44 \\
p=0.24 \quad \text { ES }=0.66\end{array}$ & $\begin{array}{l}W=2.35 \mathrm{df}=15.47 \\
p=0.03 \mathrm{ES}=0.79\end{array}$ & $M d n=3.24$ \\
\hline
\end{tabular}

Groups were compared with the Brunner-Munzel test for independent samples $(W$ : parameter of the Brunner-Munzel test, $d f:$ degrees of freedom, $p: p$ value). ES represents the effect size estimated through a measure of stochastic superiority $(.56 \leq$ small $\mathrm{ES}<.64, .64 \leq$ medium $\mathrm{ES}<.71, .71 \leq$ large $\mathrm{ES}$; see the Materials and method section).

their components. Our results showed an impact of perceptual learning on the later processing of both mixtures and single odorants. However, this effect was clearly modulated by the initial blending properties of the mixture.

Effect of experience on the perception of the $\mathrm{AB}$ blending mixture

First of all, our data supported the configural processing of the $\mathrm{AB}$ mixture, and therefore confirmed its blending status demonstrated in previous studies (in humans, Barkat et al., 2012; Le Berre et al., 2010; Le Berre et al., 2008; in rabbits,
Coureaud et al., 2011; Coureaud et al., 2009; Coureaud et al., 2008; Coureaud et al., 2014). Interestingly, we found here that the configural processing of this mixture was effective regardless of preexposure conditions, because the pineapple typicality of the mixture was higher than that of the components in all groups of subjects.

Nevertheless, significant differences in the pineapple typicality of the mixture appeared to be conditional upon preexposure, a result that highlights the influence of perceptual learning on mixture perception. Specifically, the pineapple typicality of the $\mathrm{AB}$ mixture decreased for the subjects previously exposed separately to the components A and B,

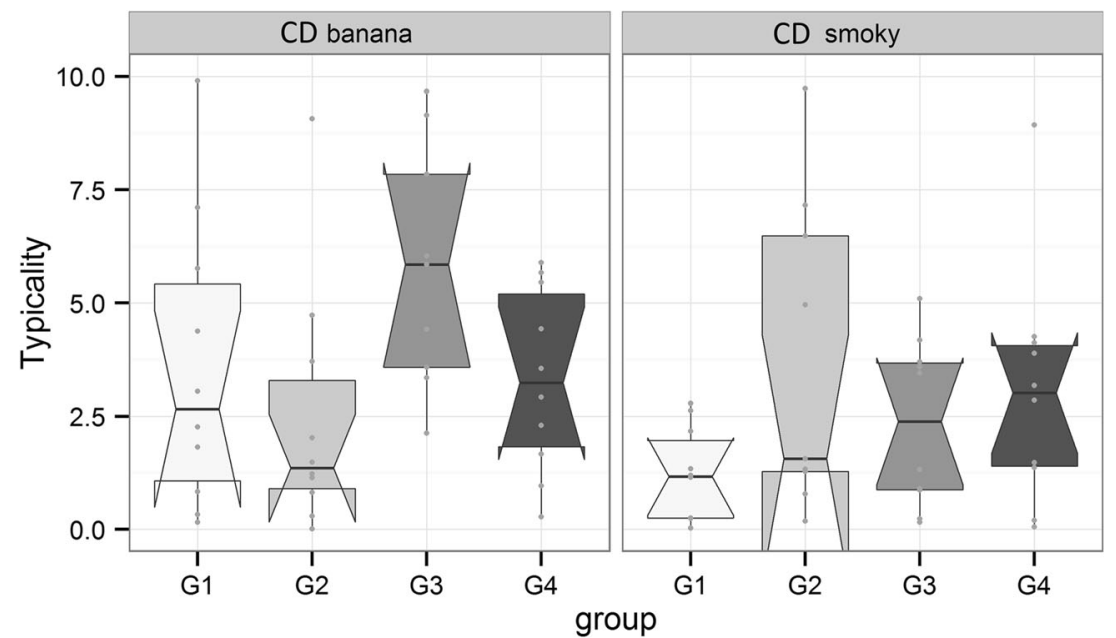

Fig. 3 Banana odor and smoky odor typicality ratings of the CD mixture, composed of $\mathrm{C}$ (guaiacol) and $\mathrm{D}$ (vanillin), evaluated by subjects preexposed to $\mathrm{AB}$ (Group $1, \mathrm{G} 1 ; N=20), \mathrm{A}$ and $\mathrm{B}(\mathrm{G} 2 ; N=19), \mathrm{CD}$ $(\mathrm{G} 3 ; N=19)$, or $\mathrm{C}$ and $\mathrm{D}(\mathrm{G} 4 ; N=20)$. The two lighter gray groups were considered controls in this analysis, because they had not been exposed to
$\mathrm{C}, \mathrm{D}$, or $\mathrm{CD}$. The black lines inside the boxes represent the medians. The upper and lower boxes represent the $75 \%$ and $25 \%$ quartiles. Dotted lines over the boxes range from a maximum of 1.5 times the interquartile distance, and notches represent $95 \%$ confidence intervals for the medians. Gray points dots represent the individual ratings of subjects 
Table 4 Congruent terms for odors: Median $(M d n)$ values of smoky (above the diagonal) and banana (below the diagonal) typicality ratings of the incongruent odorants C (smoky like odor) and D (banana like odor), evaluated by subjects preexposed to $\mathrm{AB}$ (Group $1, \mathrm{G} 1 ; N=20$ ), $\mathrm{A}$ and $\mathrm{B}$ $(\mathrm{G} 2 ; N=19), \mathrm{CD}(\mathrm{G} 3 ; N=19)$, or C and D $(\mathrm{G} 4 ; N=20)$

\begin{tabular}{|c|c|c|c|c|}
\hline D banana & G1 (AB) & $\mathbf{G 2}(\mathrm{A} \& \mathrm{~B})$ & $\mathbf{G 3}$ (CD) & G4 (C\&D) \\
\hline $\mathbf{G 1}(A B)$ & $M d n=6.38$ & & $\begin{array}{ll}W=1.01 & \mathrm{df}=14.55 \\
p=0.33 & \mathrm{ES}=0.65\end{array}$ & \\
\hline G2 (A\&B) & & $M d n=8.63$ & & $\begin{array}{ll}W=0.78 & \mathrm{df}=11.89 \\
p=0.45 & \mathrm{ES}=0.65\end{array}$ \\
\hline G3 (CD) & $\begin{array}{l}W=-0.30 \quad \mathrm{df}=10.48 \\
p=0.78 \quad \mathrm{ES}=0.51\end{array}$ & & $M d n=7.46$ & $\begin{array}{l}W=-0.34 \quad \mathrm{df}=12.70 \\
p=0.74 \quad \mathrm{ES}=0.55\end{array}$ \\
\hline G4 (C\&D) & & $\begin{array}{l}W=0.21 \quad \mathrm{df}=15.90 \\
p=0.83 \quad \text { ES }=0.53\end{array}$ & $\begin{array}{l}W=-0.60 \quad \mathrm{df}=13.04 \\
p=0.56 \quad \mathrm{ES}=0.53\end{array}$ & $M d n=8.02$ \\
\hline
\end{tabular}

Medians were compared with the Brunner-Munzel test for independent samples ( $W$ : parameter of the Brunner-Munzel test, $d f$ : degrees of freedom, $p: p$ value). ES represents the effect size estimated through a measure of stochastic superiority $(.56 \leq$ small $\mathrm{ES}<.64, .64 \leq$ medium ES $<.71, .71 \leq$ large ES; see the Materials and method section).

as compared with the subjects preexposed to unrelated odorants ( $\mathrm{C}$ and $\mathrm{D})$ or to the $\mathrm{AB}$ mixture itself. This result bolsters the hypothesis that preexposure to $\mathrm{A}$ and $\mathrm{B}$ components could decrease the configural processing of the $\mathrm{AB}$ blending mixture, an effect already observed with a ternary blending mixture in humans (Le Berre et al., 2008), and also in rabbit neonates with the same binary AB mixture (Sinding et al., 2011).

However, repeated exposure without conditioning did not seem sufficient to entail fully elemental perception of the mixture, since it was still rated as being more typical of pineapple than of its elements. Therefore, these results suggest a shift of perception from a strong configural perception toward a weak configural perception of the $\mathrm{AB}$ mixture (Kay et al., 2005; Sinding et al., 2011). In other words, the $\mathrm{AB}$ configuration might become less perceptually impenetrable, whereas the odorants become increasingly distinguishable in the mixture. This phenomenon could be the result of subjects paying increased attention to some salient attribute(s) of the mixture (Stevenson, 2001b). Indeed, perceptual learning of single components can improve olfactory quality discrimination
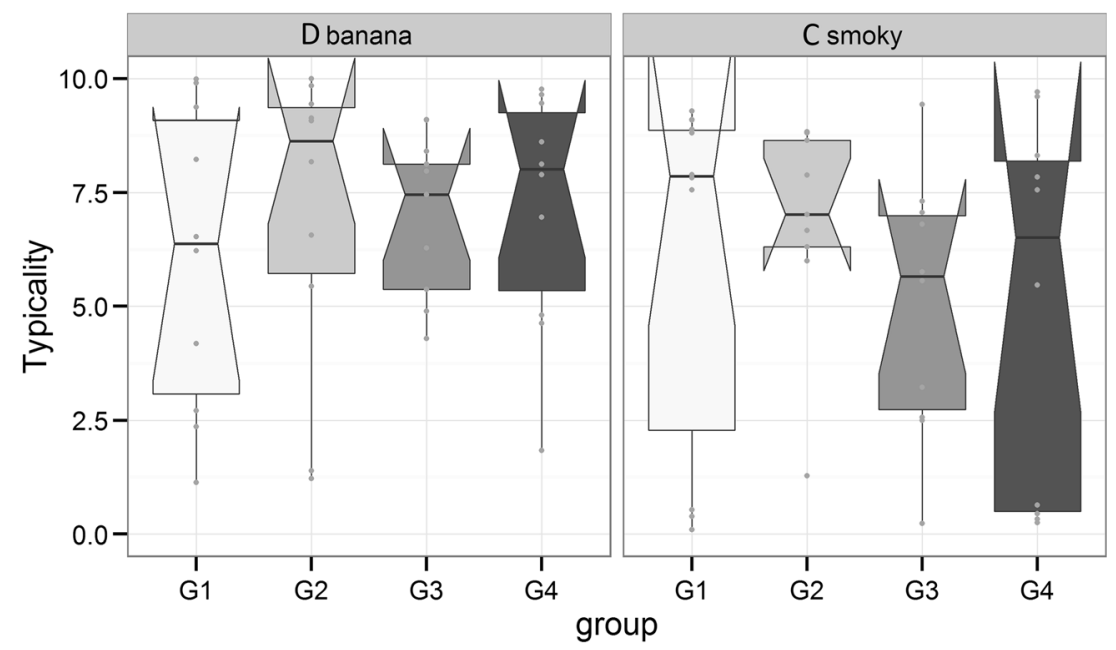

Fig. 4 Congruent terms for odors: Banana odor typicality ratings of odorant $\mathrm{D}$ and smoky odor typicality ratings of odorant $\mathrm{C}$, evaluated by subjects preexposed to $\mathrm{AB}$ (Group $1, \mathrm{G} 1 ; N=20)$, A and B (G2; $N=19)$, $\mathrm{CD}(\mathrm{G} 3 ; N=19)$, or $\mathrm{C}$ and $\mathrm{D}(\mathrm{G} 4 ; N=20)$. The two lighter gray groups were considered controls in this analysis, because they had not been exposed to $\mathrm{C}, \mathrm{D}$, or $\mathrm{CD}$. The black lines inside the boxes represent the

medians. The upper and lower boxes represent the $75 \%$ and $25 \%$ quartiles. Dotted lines over the boxes range from a maximum of 1.5 times the interquartile distance, and notches represent $95 \%$ confidence intervals for the medians. Gray points dots represent the individual ratings of subjects 
Table 5 Incongruent terms for odors: Median $(M d n)$ values of smoky (above the diagonal) and banana (below the diagonal) typicality ratings of the incongruent odorants D (banana like odor) and C (smoky like odor), evaluated by subjects preexposed to $\mathrm{AB}$ (Group $1, \mathrm{G} 1 ; N=20$ ), $\mathrm{A}$ and $\mathrm{B}$ $(\mathrm{G} 2 ; N=19), \mathrm{CD}(\mathrm{G} 3 ; N=19)$, or $\mathrm{C}$ and $\mathrm{D}(\mathrm{G} 4 ; N=20)$

\begin{tabular}{|c|c|c|c|c|}
\hline C banana & $\mathbf{G 1}(\mathrm{AB})$ & $\mathbf{G} 2$ (A\&B) & $\mathbf{G 3}$ (CD) & G4 (C\&D) \\
\hline $\mathbf{G 1}(A B)$ & $M d n=0.32$ & & $\begin{array}{l}W=-0.7 \text { df }=17.39 \\
p=0.49 \text { ES }=0.60\end{array}$ & \\
\hline G2 (A\&B) & & $M d n=0.11$ & $\begin{array}{l}W=-1.51 \quad \mathrm{df}=13.08 \\
p=0.15 \quad \text { ES }=0.63\end{array}$ & \\
\hline G3 (CD) & $\begin{array}{l}W=-1.87 \mathrm{df}=16.73 \\
p=0.08 \text { ES }=0.66\end{array}$ & $\begin{array}{l}W=-2.63 \mathrm{df}=15.39 \\
p=0.02 \mathrm{ES}=0.71\end{array}$ & $M d n=0.91$ & $\begin{array}{l}W=-0.50 \quad \mathrm{df}=13.92 \\
p=0.63 \quad \mathrm{ES}=0.57\end{array}$ \\
\hline G4 (C\&D) & & & $\begin{array}{l}W=-2.69 \quad \mathrm{df}=16.82 \\
p=0.02 \quad \mathrm{ES}=0.71\end{array}$ & $M d n=0.26$ \\
\hline
\end{tabular}

Groups were compared with the Brunner-Munzel test for independent samples $(W$ : parameter of the Brunner-Munzel test, $d f:$ degrees of freedom, $p: p$ value). ES represents the effect size estimated through a measure of stochastic superiority $(.56 \leq$ small $\mathrm{ES}<.64, .64 \leq$ medium $\mathrm{ES}<.71, .71 \leq$ large $\mathrm{ES}$; see the Materials and method section).

(Rabin, 1988). Subjects exposed to A and B components out of mixture could have perceived more distinctly the specific odors of each of the individual odorants in the mixture, in addition to the configural pineapple odor. Some neurobiological findings lend credit to this hypothesis. In adult rats, elements of a mixture became more salient after exposure, as indicated by less overlapping bulbar patterns of each component (Mandairon, Stack, \& Linster, 2006). Indeed, bulbar patterns could reflect odor identity before any higher-order modulations. Odors with similar bulbar patterns also smell more alike (Frederick, Barlas, Levins, \& Kay, 2009). The saliency of components that favor elemental processing after previous conditioning to these components has also been observed in studies with honeybees (Faber, Joerges, \& Menzel, 1999; Fernandez, Locatelli, Person-Rennell, Deleo, \& Smith, 2009) and lobsters (Derby, Hutson, Livermore, \& Lynn, 1996).

The results obtained after exposure to the $\mathrm{AB}$ mixture suggested that "experience" - at least the experience induced by the present number of exposures to $\mathrm{AB}$ - cannot improve the configural perception of the mixture. Indeed, in contrast to what we expected, we did not observe an increase of the pineapple typicality of the $\mathrm{AB}$ mixture in subjects preexposed to
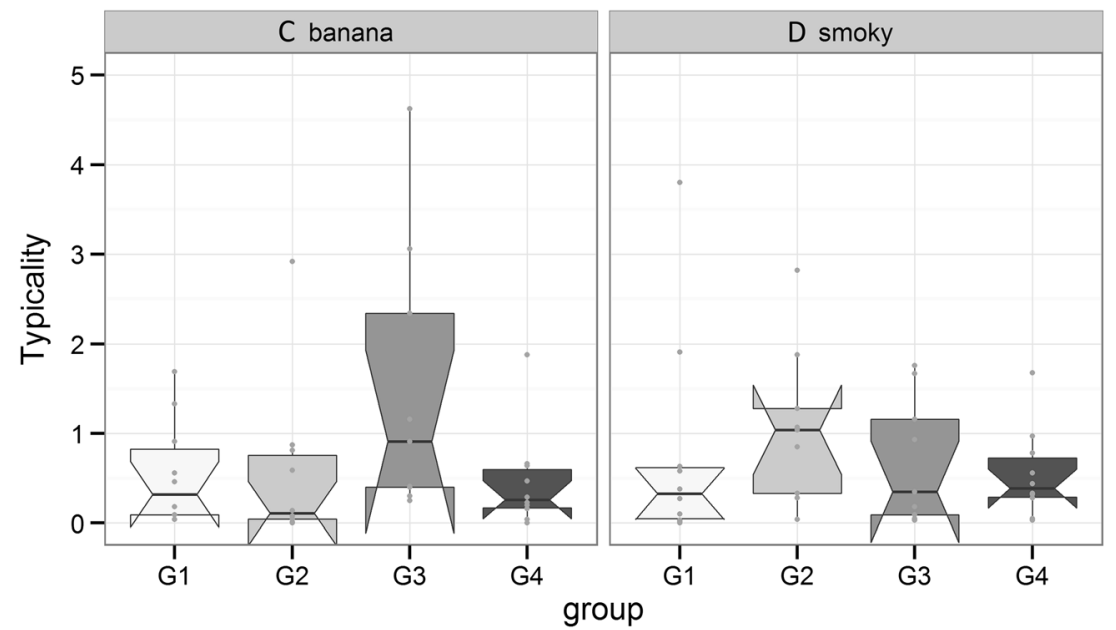

Fig. 5 Incongruent terms for odors: Banana odor typicality ratings of odorant $\mathrm{C}$ and smoky odor typicality ratings of odorant $\mathrm{D}$, evaluated by subjects preexposed to $\mathrm{AB}$ (Group $1, \mathrm{G} 1 ; N=20)$, A and B $(\mathrm{G} 2 ; N=19)$, $\mathrm{CD}(\mathrm{G} 3 ; N=19)$, or $\mathrm{C}$ and $\mathrm{D}(\mathrm{G} 4 ; N=20)$. The two lighter gray groups were considered controls in this analysis, because they had not been exposed to $\mathrm{C}, \mathrm{D}$, or $\mathrm{CD}$. The black lines inside the boxes represent the

medians. The upper and lower boxes represent the $75 \%$ and $25 \%$ quartiles. Dotted lines over the boxes range from a maximum of 1.5 times the interquartile distance, and notches represent $95 \%$ confidence intervals for the medians. Gray points dots represent the individual ratings of subjects 
AB (G1), as compared with groups preexposed to the A and B components separately (G2) or to another mixture (CD). Neither did we observe any significant influence of mere exposure to $\mathrm{AB}$ on the pineapple typicality of the single A and $\mathrm{B}$ components. Nevertheless, it is worth noting that the pineapple typicality of $\mathrm{AB}$ did not decrease, which suggests that the configural perception is still favored in the subjects preexposed to the mixture. Several reasons could explain the lack of improvement in experience-induced configural processing of the mixture. First, the way in which subjects are preexposed and the "strength" of the preexposure may be involved. The amount of preexposure to $\mathrm{AB}$ may be insufficient to enhance its configural processing. Indeed, in a study using newborn rabbits, such an improvement in configural processing only emerged after nine repeated conditionings to the $\mathrm{AB}$ mixture, which was initially processed as weakly configural by the pups (Sinding et al., 2011). The context of this preexposure could additionally modulate the strength of the encoded concepts. In the case of rabbit pups, the mammary pheromone was used as a reinforcer during associative conditioning to the odor mixture (Coureaud et al., 2009; Coureaud et al., 2008; Coureaud et al., 2014), which was likely to produce strong conditioning due to the critical biological value of this natural and maternal odor signal for neonates (Coureaud et al., 2010; Coureaud et al., 2006). In contrast, the preexposure of human subjects consisted of a mere exposure repeated twice, without pairing with any reinforcing agent. Second, one cannot exclude that a developmental effect may contribute to the relative plasticity of the $A B$ configural perception observed in newborn rabbits but not in human adults. Third, the task of typicality rating may limit the proper visualization of a higher configural perception, since a scale edge effect may have inflected our results. Indeed, to be rated as more configural, the $\mathrm{AB}$ mixture should have obtained a typicality score greater than 8 (maximum median value observed here, 10 being the possible maximum value), which is unlikely. This methodological issue might be overcome by using complementary methods, such as a similarity test or a free-sorting task (see Sinding et al., 2013, for tests of a sixcomponent mixture). A free-sorting task would allow for assessing the similarity between the mixture and its components, and we could expect that the group preexposed to the mixture would consider the mixture more distant perceptually from its components than control groups would.

Effect of experience on the perception of the CD nonblending mixture

The results obtained with the $\mathrm{CD}$ nonblending mixture were used to assess whether experience influences the processing of a mixture that is initially processed elementally (i.e., that carries both the banana and smoky odors). First, preexposure to the CD mixture had a clear effect on its later perception. However, contrary to our initial assumption, the CD perception did not become strictly configural, since perception of the smoky odor decreased while that of the banana odor increased. Nevertheless, interestingly, subjects found that after preexposure to the $\mathrm{CD}$ mixture, the single odorant $\mathrm{C}$ carried a light banana odor, although its initial quality was typically smoky. This transfer of quality between the paired components was not found after separate preexposures to $\mathrm{C}$ and to D. Such quality transfer has already been shown after repeated exposures to cherry/smoky or lemon/smoky binary mixtures, resulting in subjects later evaluating the cherry or lemon odors as also being smoky (Stevenson, 2001a). According to Stevenson (2001a), this transfer of quality between paired odorants could result from an association between the mixture's elements, which is reconcilable with configural processing of the mixture induced by coexposure and learning. Therefore, one may wonder whether the experience-induced transfer of quality from D to C noted in the present study is a form of weak configural processing of the $\mathrm{CD}$ mixture. Experience-induced transfer of qualities between stimuli has also been described when pairing odors and tastes. In that case, an odor can acquire the perceptual properties of a taste through the formation of a configuration (flavor) from the smell and taste elements (Prescott \& Murphy, 2009). The configuration is learned and is stored in the brain as a specific pattern that is not independent of its elements and could be reactivated by each of them (Small \& Prescott, 2005).

\section{Conclusion}

We determined here that both elemental and configural perceptions can be modulated by experience without conditioning in humans. However, the type of mixture processing (i.e., whether it is initially blending or not) strongly modulates the effect of experience. Our results suggest that both elemental and configural perceptions operate along a continuum and rely on rather independent but nonexclusive processes. On the basis of the results reported here, and with the help of similar odor models and experimental designs, one could attempt to localize the neural substrates of configural and elemental perceptions in humans.

Author note The authors thank John Prescott for his comments on a previous version of the manuscript, and an anonymous reviewer for valuable input as to the statistical analyses. The work was supported by grants from the Burgundy Regional Council and EU-ERDF to G.C. and T.T.D., and by a fellowship from the French MESR to C.S.

\section{References}

Atanasova, B., Langlois, D., Nicklaus, S., Chabanet, C., \& Etiévant, P. (2004). Evaluation of olfactory intensity: Comparative study of two methods. Journal of Sensory Studies, 19, 307-326. doi:10.1111/j. 1745-459X.2004.tb00150.x 
Atanasova, B., Thomas-Danguin, T., Chabanet, C., Langlois, D., Nicklaus, S., \& Etiévant, P. (2005). Perceptual interactions in odour mixtures: Odour quality in binary mixtures of woody and fruity wine odorants. Chemical Senses, 30, 209-217. doi:10.1093/chemse/ bji016

Barkat, S., Le Berre, E., Coureaud, G., Sicard, G., \& Thomas-Danguin, T. (2012). Perceptual blending in odor mixtures depends on the nature of odorants and human olfactory expertise. Chemical Senses, 37, 159-166. doi:10.1093/chemse/bjr086

Brunner, E., \& Munzel, U. (2000). The nonparametric Behrens-Fisher problem: Asymptotic theory and a small-sample approximation. Biometrical Journal, 42(1), 17-25. doi:10.1002/(sici)15214036(200001)42:1<17::aid-bimj17>3.0.co;2-u

Bushdid, C., Magnasco, M. O., Vosshall, L. B., \& Keller, A. (2014). Humans can discriminate more than 1 trillion olfactory stimuli. Science, 343, 1370-1372. doi:10.1126/science.1249168

Cain, W. S., \& Gent, J. F. (1991). Olfactory sensitivity: Reliability, generality, and association with aging. Journal of Experimental Psychology: Human Perception and Performance, 17, 382-391. doi:10.1037/0096-1523.17.2.382

Case, T. I., Stevenson, R. J., \& Dempsey, R. A. (2004). Reduced discriminability following perceptual learning with odours. Perception, 33, 113-119. doi:10.1068/p5044

Chrea, C., Valentin, D., Sulmont-Rosse, C., Mai, H. L., Nguyen, D. H., \& Abdi, H. (2004). Culture and odor categorization: Agreement between cultures depends upon the odors. Food Quality and Preference, 15, 669-679. doi:10.1016/j.foodqual.2003.10.005

Coureaud, G., Charra, R., Datiche, F., Sinding, C., Thomas-Danguin, T., Languille, S., \& Schaal, B. (2010). A pheromone to behave, a pheromone to learn: The rabbit mammary pheromone. Journal of Comparative Physiology A, 196, 779-790. doi:10.1007/s00359010-0548-y

Coureaud, G., Gibaud, D., Le Berre, E., Schaal, B., \& Thomas-Danguin, T. (2011). Proportion of odorants impacts the configural versus elemental perception of a binary blending mixture in newborn rabbits. Chemical Senses, 36, 693-700. doi:10.1093/chemse/bjr049

Coureaud, G., Hamdani, Y., Schaal, B., \& Thomas-Danguin, T. (2009). Elemental and configural processing of odour mixtures in the newborn rabbit. Journal of Experimental Biology, 212, 2525-2531. doi: $10.1242 /$ jeb. 032235

Coureaud, G., Moncomble, A.-S., Montigny, D., Dewas, M., Perrier, G., \& Schaal, B. (2006). A pheromone that rapidly promotes learning in the newborn. Current Biology, 16, 1956-1961. doi:10.1016/j.cub. 2006.08.030

Coureaud, G., Thomas-Danguin, T., Le Berre, E., \& Schaal, B. (2008). Perception of odor blending mixtures in the newborn rabbit. Physiology and Behavior, 95, 194-199. doi:10.1016/j.physbeh. 2008.05.018

Coureaud, G., Thomas-Danguin, T., Wilson, D. A., \& Ferreira, G. (2014). Neonatal representation of odour objects: Distinct memories of the whole and its parts. Proceedings of the Royal Society B, 281, 20133319. doi:10.1098/rspb.2013.3319

Derby, C. D., Hutson, M., Livermore, A., \& Lynn, W. H. (1996). Generalization among related complex odorant mixtures and their components: Analysis of olfactory perception in the spiny lobster. Physiology \& Behavior, 60, 87-95. doi:10.1016/0031-9384(95) 02237-6

Doty, R. L. (2001). Olfaction. Annual Review of Psychology, 52, 423452. doi:10.1146/annurev.psych.52.1.423

Faber, T., Joerges, J., \& Menzel, R. (1999). Associative learning modifies neural representations of odors in the insect brain. Nature Neuroscience, 2, 74-78. doi:10.1038/4576

Fernandez, P. C., Locatelli, F. F., Person-Rennell, N., Deleo, G., \& Smith, B. H. (2009). Associative conditioning tunes transient dynamics of early olfactory processing. Journal of Neuroscience, 29, 1019110202. doi:10.1523/JNEUROSCI. 1874-09.2009
Frederick, D. E., Barlas, L., Levins, A., \& Kay, L. M. (2009). A critical test of the overlap hypothesis for odor mixture perception. Behavioral Neuroscience, 123, 430-437. doi:10.1037/a0014729

Kay, L. M., Crk, T., \& Thorngate, J. (2005). A redefinition of odor mixture quality. Behavioral Neuroscience, 119, 726-733. doi:10.1037/ 0735-7044.119.3.726

Le Berre, E., Jarmuzek, E., Béno, N., Etiévant, P., Prescott, J., \& ThomasDanguin, T. (2010). Learning influences the perception of odor mixtures. Chemosensory Perception, 3, 156-166. doi:10.1007/s12078010-9076-y

Le Berre, E., Thomas-Danguin, T., Beno, N., Coureaud, G., Etievant, P., \& Prescott, J. (2008). Perceptual processing strategy and exposure influence the perception of odor mixtures. Chemical Senses, 33, 193-199. doi:10.1093/chemse/bjm080

Lesschaeve, I., \& Issanchou, S. (1996). Could selection tests detect the future performance of descriptive panellists? Food Quality and Preference, 7, 177-183. doi:10.1016/S0950-3293(96)00022-5

Livermore, A., Hutson, M., Ngo, V., Hadjisimos, R., \& Derby, C. D. (1997). Elemental and configural learning and the perception of odorant mixtures by the spiny lobster Panulirus Argus. Physiology and Behavior, 62, 169-174. doi:10.1016/S0031-9384(97)00031-0

Livermore, A., \& Laing, D. G. (1996). Influence of training and experience on the perception of multicomponent odor mixtures. Journal of Experimental Psychology: Human Perception and Performance, 22, 267-277. doi:10.1037/0096-1523.22.2.267

Livermore, A., \& Laing, D. G. (1998). The influence of chemical complexity on the perception of multicomponent odor mixtures. Perception \& Psychophysics, 60, 650-661. doi:10.3758/ BF03206052

Mandairon, N., Stack, C., \& Linster, C. (2006). Olfactory enrichment improves the recognition of individual components in mixtures. Physiology and Behavior, 89, 379-384. doi:10.1016/j.physbeh. 2006.07.013

Marmolejo-Ramos, F., Elosúa, M. R., Yamada, Y., Hamm, N. F., \& Noguchi, K. (2013). Appraisal of space words and allocation of emotion words in bodily space. PLoS ONE, 8, e81688. doi:10. 1371/journal.pone.0081688

Morris, J. D. (1995). SAM: The Self-Assessment Manikin—An efficient cross-cultural measurement of emotional response. Journal of Advertising Research, 35, 63-68.

Neuhäuser, M., \& Ruxton, G. D. (2009). Distribution-free two-sample comparisons in the case of heterogeneous variances. Behavioral Ecology and Sociobiology, 63, 617-623. doi:10.1007/s00265-0080683-4

Ouss, L., Carton, S., Jouvent, R., \& Widloêcher, D. (1990). Traduction et validation de l'échelle $d$ 'émotions différentielle d'Izard: Exploration de la qualification verbale des émotions. L'Encéphale, 16, 453-458.

Prescott, J., \& Murphy, S. (2009). Inhibition of evaluative and perceptual odour-taste learning by attention to the stimulus elements. Quarterly Journal of Experimental Psychology, 62, 2133-2140. doi:10.1080/17470210903031169

Rabin, M. D. (1988). Experience facilitates olfactory quality discrimination. Perception \& Psychophysics, 44, 532-540. doi:10.3758/ BF03207487

Rouby, C., Thomas-Danguin, T., Sicard, G., Vigouroux, M., Jiang, T., Poitevineau, J., \& Issanchou, S. (2005). Influence du contexte sémantique sur la performance d'identification d'odeurs. Psychologie Française, 50, 225-239. doi:10.1016/j.psfr.2004.11. 003

Sinding, C., Thomas-Danguin, T., Chambault, A., Béno, N., Dosne, T., Chabanet, C., \& Coureaud, G. (2013). Rabbit neonates and human adults perceive a blending 6-component odor mixture in a comparable manner. PLoS ONE, 8, e53534. doi:10.1371/journal.pone. 0053534

Sinding, C., Thomas-Danguin, T., Crepeaux, G., Schaal, B., \& Coureaud, G. (2011). Experience influences elemental and configural 
perception of certain binary odour mixtures in newborn rabbits. Journal of Experimental Biology, 214, 4171-4178. doi:10.1242/ Jeb.063610

Small, D. M., \& Prescott, J. (2005). Odor/taste integration and the perception of flavor. Experimental Brain Research, 166, 345-357. doi: 10.1007/s00221-005-2376-9

Staubli, U., Fraser, D., Faraday, R., \& Lynch, G. (1987). Olfaction and the "data" memory system in rats. Behavioral Neuroscience, 101, 757765. doi:10.1037/0735-7044.101.6.757

Stevenson, R. J. (2001a). Associative learning and odor quality perception: How sniffing an odor mixture can alter the smell of its parts. Learning and Motivation, 32, 154-177. doi:10.1006/lmot.2000. 1070

Stevenson, R. J. (2001b). Is sweetness taste enhancement cognitively impenetrable? Effects of exposure, training and knowledge. Appetite, 36, 241-242. doi:10.1006/appe.2001.0401

Stevenson, R. J. (2001c). Perceptual learning with odors: Implications for psychological accounts of odor quality perception. Psychonomic Bulletin \& Review, 8, 708-712. doi:10. 3758/BF03196207

Stevenson, R. J., \& Wilson, D. A. (2007). Odour perception: An objectrecognition approach. Perception, 36, 1821-1833. doi:10.1068/ p5563
Sulmont-Rossé, C., Issanchou, S., \& Köster, E. P. (2005). Odor naming methodology: Correct identification with multiple-choice versus repeatable identification in a free task. Chemical Senses, 30, $23-27$. doi:10.1093/chemse/bjh252

Thomas-Danguin, T., Rouby, C., Sicard, G., Vigouroux, M., Farget, V., Johanson, A., \& Dumont, J. P. (2003). Development of the ETOC: A European test of olfactory capabilities. Rhinology, 41, 142-151.

Thomas-Danguin, T., Sinding, C., Romagny, S., El Mountassir, F., Atanasova, B., Le Berre, E., \& Coureaud, G. (2014). The perception of odor objects in everyday life: A review on the processing of odor mixtures. Frontiers in Psychology, 5, 504. doi:10.3389/fpsyg.2014. 00504

Valentincic, T., Kralj, J., Stenovec, M., Koce, A., \& Caprio, J. (2000). The behavioral detection of binary mixtures of amino acids and their individual components by catfish. Journal of Experimental Biology, 203, 3307-3317. Retrieved from http://jeb.biologists.org/ cgi/content/abstract/203/21/3307

Wilson, D. A. (2003). Rapid, experience-induced enhancement in odorant discrimination by anterior piriform cortex neurons. Journal of Neurophysiology, 90(1), 65-72. doi:10.1152/jn.00133.2003

Wilson, D. A., \& Stevenson, R. J. (2003). The fundamental role of memory in olfactory perception. Trends in Neurosciences, 26, 243-247. doi:10.1016/S0166-2236(03)00076-6 